\title{
IGR J17252-3616: an accreting pulsar observed by INTEGRAL and XMM-Newton
}

\author{
J. A. Zurita Heras ${ }^{1,2} \dagger$, G. de Cesare $^{3}$, R. Walter ${ }^{1,2}$, A. Bodaghee ${ }^{1,2}$, \\ G. Bélanger ${ }^{4}$, T. J.-L. Courvoisier ${ }^{1,2}$, S. E. Shaw ${ }^{5,1}$ and J. B. Stephen ${ }^{6}$ \\ ${ }^{1}$ INTEGRAL Science Data Centre, Versoix, Switzerland \\ email: Juan.Zurita@obs.unige.ch \\ ${ }^{2}$ Observatoire de Genève, Sauverny, Switzerland \\ ${ }^{3}$ IASF-INAF, Via Fosso del Cavaliere 100, 00133 Roma, Italy \\ ${ }^{4}$ Service d'Astrophysique, DAPNIA/DSM/CEA, 91191 Gif-sur-Yvette, France \\ ${ }^{5}$ School of Physics and Astronomy, University of Southampton, Highfield, SO171BJ, UK \\ ${ }^{6} \mathrm{IASF} / \mathrm{CNR}$, Via Piero Gobetti 101, 40129 Bologna, Italy
}

\begin{abstract}
IGR J17252-3616 is the hard X-ray counterpart of EXO 1722-363. The regular monitoring by INTEGRAL shows that IGR J17252-3616 is a persistent source with an average count rate of $\sim 6.4 \mathrm{mCrab}$ in the $20-60 \mathrm{keV}$ energy band. A follow-up observation with XMM-Newton showed that the source is located at R.A. $(2000.0)=17^{h} 25^{m} 11.4^{s}$ and Dec. $=-36^{\circ} 16^{\prime} 58.6^{\prime \prime}$ with an uncertainty of $4^{\prime \prime}$.

The source is a binary X-ray pulsar with a spin period of $413.7 \mathrm{~s}$. The spectral shape is typical for an accreting pulsar except that a huge intrinsic absorption and a cold iron fluorescence line are detected. The absorbing column density and cold iron line do not vary with the pulse period. The observations suggest that the source is a wind-fed accreting pulsar accompanied by a supergiant star.
\end{abstract}

Keywords. X-rays: binaries, X-rays: individual: IGR J17252-3616 = EXO 1722-363.

\section{Introduction}

EXO 1722-363 was discovered by EXOSAT in June 1984 (Warwick, Norton, Turner, et al. 1988). From Ginga observations in 1987 and 1988, Tawara, Yamauchi, Awaki, et al. (1989) and Takeuchi, Koyama \& Warwick (1990) detected a pulsation of $413.9 \mathrm{~s}$, important variations of the intensity in X-rays, a hard spectrum with important lowenergy absorption and an emission line at $6.2 \mathrm{keV}$. Corbet, Markwardt \& Swank (2005) resolved the orbital period of 9.741 days and detected a varying high column density with RXTE data. These investigations conclude that the system is a high mass X-ray binary (HMXB).

\section{Observations \& Analysis}

INTEGRAL is a hard X-ray and $\gamma$-ray observatory of the European Space Agency (ESA). A total exposure of 6.5 Ms was accumulated between MJD 52671 and 53294. A follow-up observation with XMM-Newton was performed on March 21, 2004, for three hours (MJD 53085.542-53085.667).

We focused our work on INTEGRAL IBIS/ISGRI and XMM-Newton EPIC instruments. The data were reduced with OSA 4.2 and SAS 6.1.0. Images, light curves and spectra were generated. For ISGRI, a detailed timing and spectral analysis was performed

$\dagger$ Present address: ISDC, ch. d'Ecogia 16, 1290 Versoix, Switzerland. 

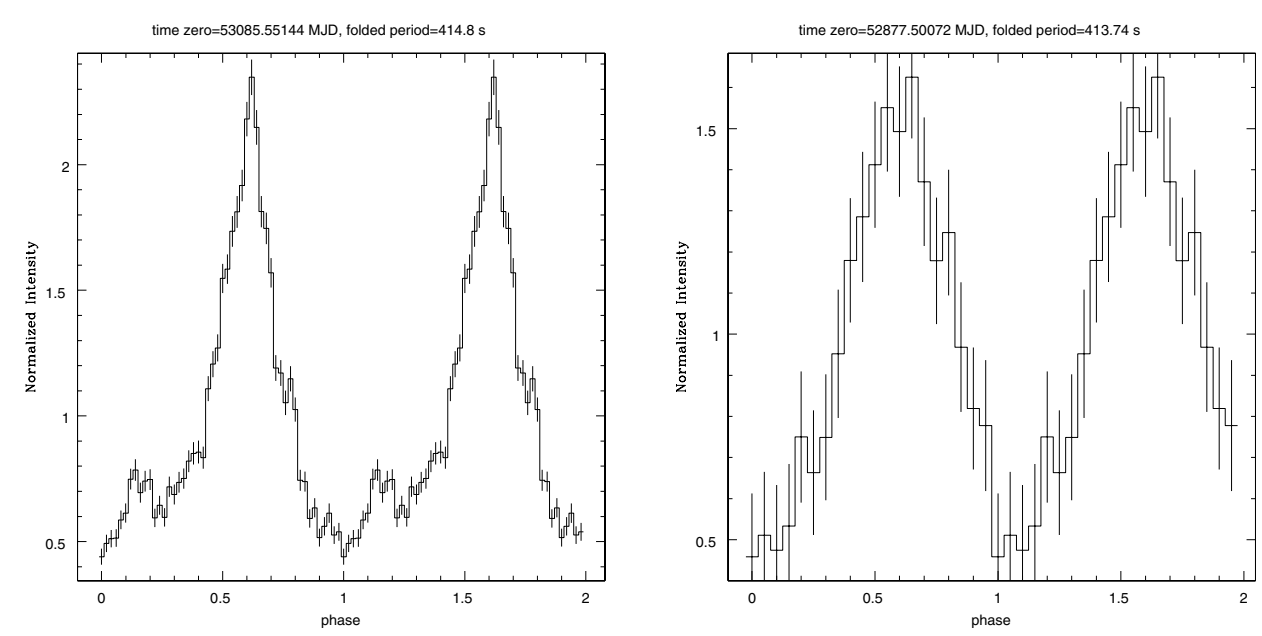

Figure 1. Folded light curves. left: pn, 0.5-10 keV; right: ISGRI, 20-60 keV.
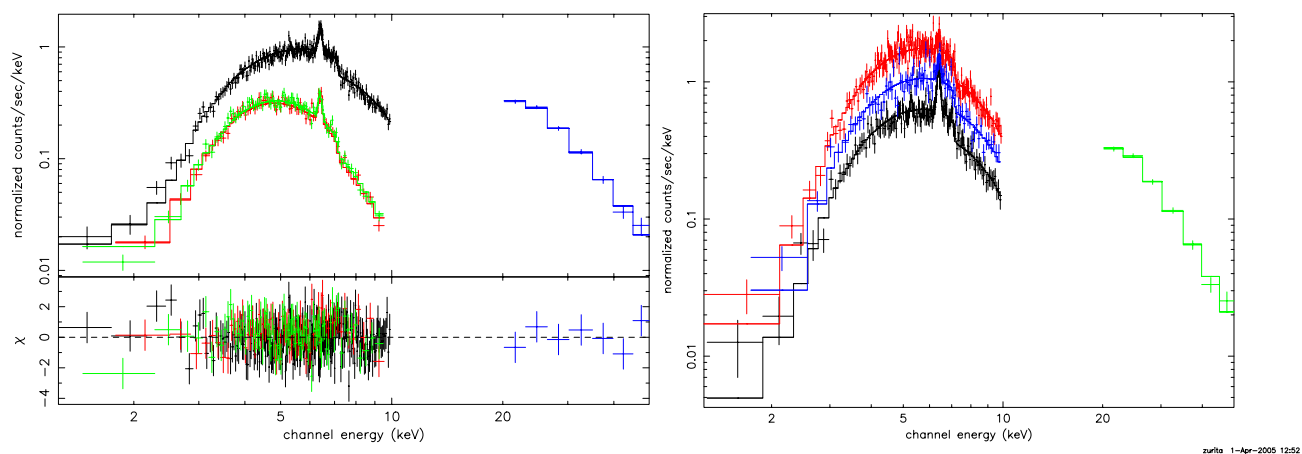

Figure 2. Combined EPIC+ISGRI spectra. The ISGRI spectrum is in the hard X-ray band. left: Average pn and MOS[12] spectra; right: Phase-resolved spectra for pn.

on revolution 106 (MJD 52877.4-52880.4) where the source reached its maximum flux. A pulsation has been detected in both pn and ISGRI data of $415 \pm 5 \mathrm{~s}$ and $413.7 \pm 0.4 \mathrm{~s}$, respectively (see Fig. 1). The spectrum can be fitted with a flat power law plus an energy cutoff $\left(\Gamma \sim 0.02, E_{\mathrm{c}} \sim 8.2 \mathrm{keV}, \chi^{2} /\right.$ d.o.f.=401/376) or with a comptonized model $\left(k T_{\mathrm{e}} \sim 5.5 \mathrm{keV}, \tau \sim 7.8, \chi^{2} /\right.$ d.o.f. $=401 / 376$, see Fig. 2 left $)$. The spectrum also indicates a large hydrogen column density of $N_{\mathrm{H}} \sim 1510^{22}$ atoms $\mathrm{cm}^{-2}$ suggesting an intrinsic absorption. The Fe $\mathrm{K} \alpha$ line at $6.4 \mathrm{keV}$ is clearly detected. Phase-resolved spectroscopy does not show any variation in the continuum except the total emitted flux (see Fig. 2 right). The absorption is constant along the pulse phase.

The observed features of IGR J17252-3616 clearly indicate that it is the hard X-ray counterpart of EXO 1722-363 even if the positions are not compatible.

\section{References}

Corbet, R. H. D., Markwardt, C. B., \& Swank, J. H. 2005, ApJ in press

Takeuchi, Y., Koyama, K., \& Warwick, R. S. 1990, PASJ 42, 287

Tawara, Y., Yamauchi, S., Awaki, H., Kii, T., Koyama, K., \& Nagase, F. 1989, PASJ 41, 473

Warwick, R. S., Norton, A. J., Turner, M. J. L., Watson, M. G. \& Willingale, R. 1988, MNRAS 232,551 